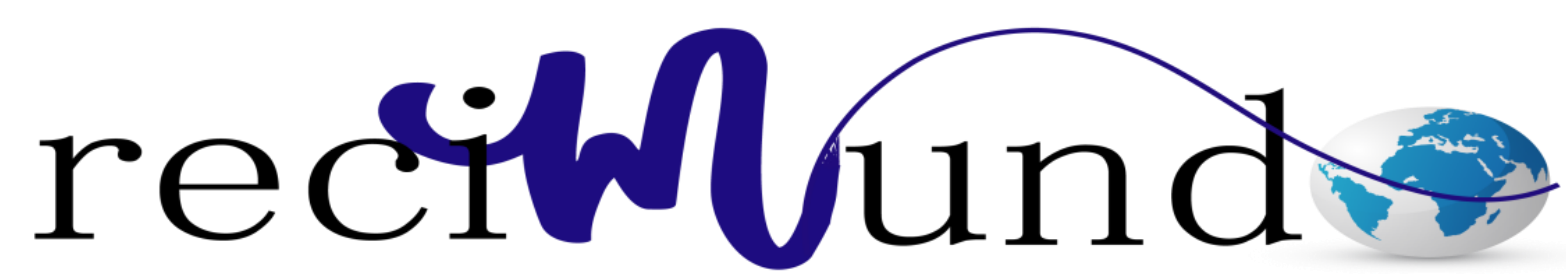

Revista Científica Mundo de la Investígación y el Conocimiento

David Israel Cárdenas Acosta ${ }^{\text {a }}$ S Sandra Elizabeth Montufar Paguay ${ }^{\text {b }}$

Indicaciones de Orquidopexia en problemas testiculares en recién nacidos

Indications of Orchidopexy in testicular problems in newborns

Revista Científica Mundo de la Investigación y el Conocimiento. Vol. 3 núm.3. Esp., noviembre, ISSN: 2588-073X, 2019, pp. 146-158

DOI: $10.26820 /$ recimundo/3.(3.Esp).noviembre.2019.146-158

URL: http://recimundo.com/index.php/es/article/view/594

Código UNESCO: 3205 Medicina Interna

Tipo de Investigación: Artículo de Revisión

(C) RECIMUNDO; Editorial Saberes del Conocimiento, 2019

Recibido: 15/09/2019

Aceptado: 23/10/2019

Publicado: 30/11/2019

Correspondencia: me.israel.cardenas@gmail.com

a. Médico General; Investigador Independiente; Guayaquil, Ecuador; me.israel.cardenas@ gmail.com

b. Médico General; Investigadora Independiente; Guayaquil, Ecuador; sandramontufar@rocketmail.com 


\section{Indicaciones de Orquidopexia en problemas testiculares en recién nacidos}

Vol. 3, núm. 3 Esp., (2019)

David Israel Cárdenas Acosta; Sandra Elizabeth Montufar Paguay

\section{RESUMEN}

Hoy en día la indicación para la Orquidopexia en pacientes con criptorquidia mayor de un año de

edad, es la reducción del riesgo de torsión, facilita la exploración del testículo, sobre todo si se presenta un tumor testicular, mejora la función endocrina del mismo y crea un escroto con apariencia normal. La criptorquidia es la falta del descenso testicular (TND). La metodología de la investigación es de tipo revisión bibliográfica. Las principales conclusiones indican que esta afección se puede generar durante el término de la gestación y que no puede pasar mucho tiempo para ser corregida por que pone en riesgo la zona testicular, aparte que su corrección preserva la función reproductiva y hormonal del testículo, se recomienda la laparoscopia para corregir esta malformación, ya que los procedimientos de abertura total pueden generar mayores complicaciones. Básicamente la intervención quirúrgica lo que hace es descender el testículo hasta la bolsa escrotal.

Palabras clave: Criptorquidia; Orquidopexia; Testículo; Escroto; Laparoscopia. 


\section{Indicaciones de Orquidopexia en problemas testiculares en recién nacidos}

Vol. 3, núm. 3 Esp., (2019)

David Israel Cárdenas Acosta; Sandra Elizabeth Montufar Paguay

\section{ABSTRACT}

Nowadays the indication for Orquidopexia in patients with cryptorchidism older than one year of age, is the reduction of the risk of torsion, facilitates the examination of the testicle, especially if a testicular tumor occurs, improves its endocrine function and creates A scrotum with normal appearance. Cryptorchidism is the lack of testicular descent (TND). The research methodology is a bibliographic review type. The main conclusions indicate that this condition can be generated during the term of pregnancy and that it may not take long to be corrected because it puts the testicular area at risk, apart from the fact that its correction preserves the reproductive and hormonal function of the testis, it is recommended laparoscopy to correct this malformation, since the procedures of total opening can generate major complications. Basically the surgical intervention what it does is lower the testicle to the scrotal bag.

Keywords: Cryptorchidism; Orchidopexy; Testis; Scrotum; Laparoscopy. 


\section{Indicaciones de Orquidopexia en problemas testiculares en recién nacidos}

Vol. 3, núm. 3 Esp., (2019)

David Israel Cárdenas Acosta; Sandra Elizabeth Montufar Paguay

\section{Introducción.}

El testículo no descendido (TND) se diagnostica desde el nacimiento o en las primeras consultas con el pediatra; se considera la malformación urogenital más frecuente en el varón. La revisión del área inguinal en búsqueda del testículo no siempre es sencilla y exige la intervención de un médico pediatra experimentado; en consecuencia, cuando no puede palparse, debe consultarse al urólogo pediatra. Esta anomalía se presenta en el 3\% al 5\% de los recién nacidos y en el $0.8 \%$ de los lactantes después de los 10 meses de edad. En la mayor parte de los casos, el descenso tiene lugar en los primeros tres meses de edad (Riquelme Heras, Landa Juárez, Miguel Gómez, Meneses Juárez, \& Andraca Dumit, 2009, pág. 216).

\section{Imagen 1. Testículos no descendidos}

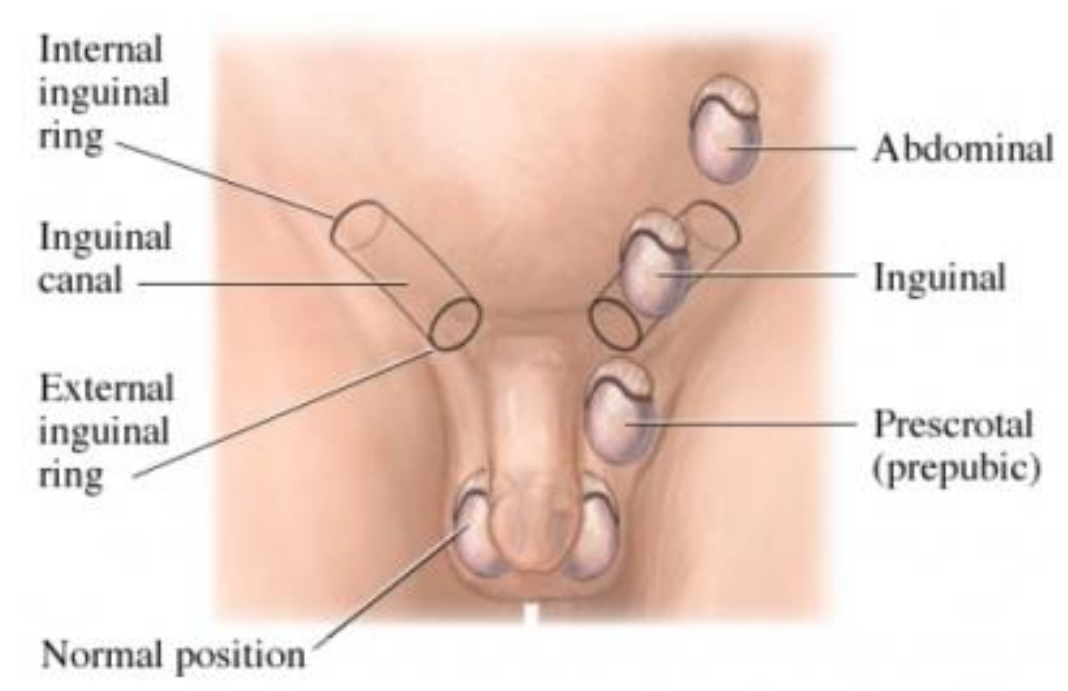

Fuente: (Neff, s.f.).

A la falta de descenso testicular se le conoce como Criptorquidia, la cual tiene gran importancia clínica dada sus implicaciones tanto en la fecundidad como en el desarrollo de 


\section{Indicaciones de Orquidopexia en problemas testiculares en recién nacidos}

Vol. 3, núm. 3 Esp., (2019)

David Israel Cárdenas Acosta; Sandra Elizabeth Montufar Paguay

tumores malignos. Hoy en día la indicación para la Orquidopexia en pacientes con criptorquidia mayor de un año de edad, es la reducción del riesgo de torsión, facilita la exploración del testículo, sobre todo si se presenta un tumor testicular, mejora la función endocrina del mismo y crea un escroto con apariencia normal (Herrera García, Cuevas Alpuche, Solorzano Morales, Aguilar Anzures, \& Olivera Vasquez, 2008, pág. 162).

Generalmente, los testículos se ubican en la bolsa escrotal durante el período de gestación. En este periodo, los testículos se desarrollan y descienden desde cavidad abdominal, hacia la bolsa escrotal, por medio de los canales inguinales. Si bien la criptorquidia no tiene ninguna sintomatología específica que oriente el cuadro clínico, el médico deberá estar atento al descenso de ambos testículos, desde el nacimiento del niño hasta cumplir el año de vida (Chocho Tapia, 2013, pág. 10).

La criptorquidia afecta al $4 \%$ de los varones recién nacidos. Aproximadamente de $70 \%$ a 77\% de los testículos criptorquídicos desciende espontáneamente a los 3 meses de edad. El bajo peso al nacer puede ser el factor principal de la criptorquidia al nacer y durante el primer año de vida, independientemente de la duración de la gestación. El 80\% de los testículos no descendidos son palpables y el restante $20 \%$ no se palpa. El descenso testicular se completa normalmente entre 30 y 32 semanas de gestación (Herrera-García, Cuevas-Alpuche, Olivera-Vázquez, Aguilar-Anzures, \& Granados-Navas, 2009, pág. 89).

La criptorquidia ha aumentado en los últimos 20 años, por mejor reconocimiento de la patología y mayor número de niños prematuros que sobreviven después del nacimiento. La clasificación más utilizada lo divide en palpable o no palpable y, por su localización, en aquel que detuvo su descenso en alguna parte del trayecto normal o en testículo ectópico verdadero, 


\section{Indicaciones de Orquidopexia en problemas testiculares en recién nacidos}

Vol. 3, núm. 3 Esp., (2019)

David Israel Cárdenas Acosta; Sandra Elizabeth Montufar Paguay

cuando su descenso se localiza fuera del trayecto normal; este puede encontrarse en la región perineal, conducto femoral, área púbica próxima al pene e incluso en hemiescroto contralateral (Córdova, Marín, Sánchez-Paredes, \& Borbolla-Sala, 2013).

Imagen 2. Bolsa escrotal y testículo derecho

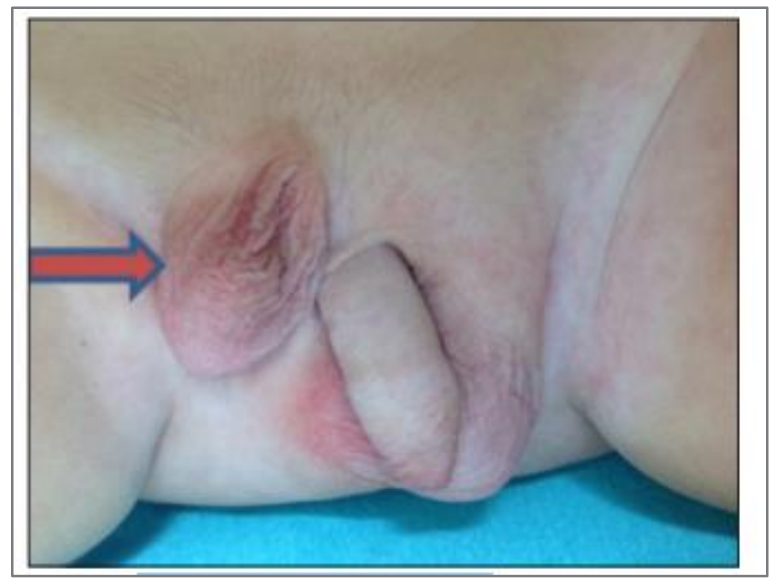

Fuente: (Córdova, Marín, Sánchez-Paredes, \& Borbolla-Sala, 2013). 


\section{Indicaciones de Orquidopexia en problemas testiculares en recién nacidos}

Vol. 3, núm. 3 Esp., (2019)

David Israel Cárdenas Acosta; Sandra Elizabeth Montufar Paguay

Imagen 3. Orquidopexia y realización de colgajo

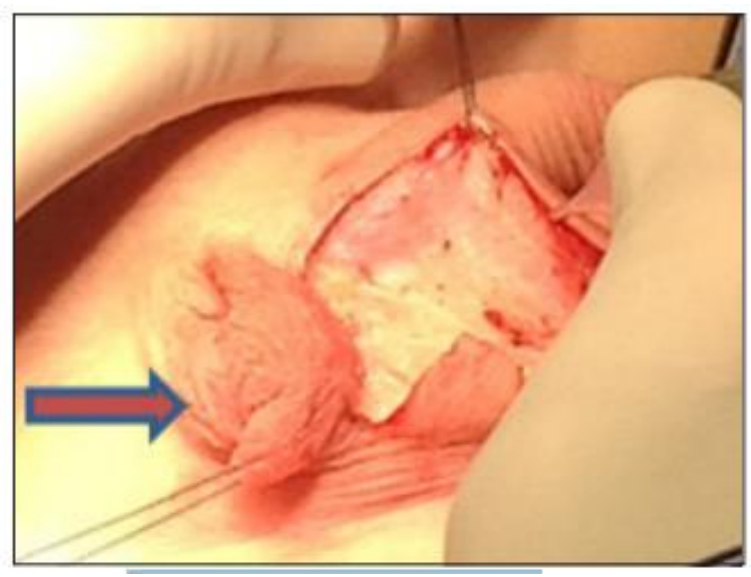

Fuente: (Córdova, Marín, Sánchez-Paredes, \& Borbolla-Sala, 2013).

Imagen 4. Testículo y bolsa escrotal en posición anatómica normal

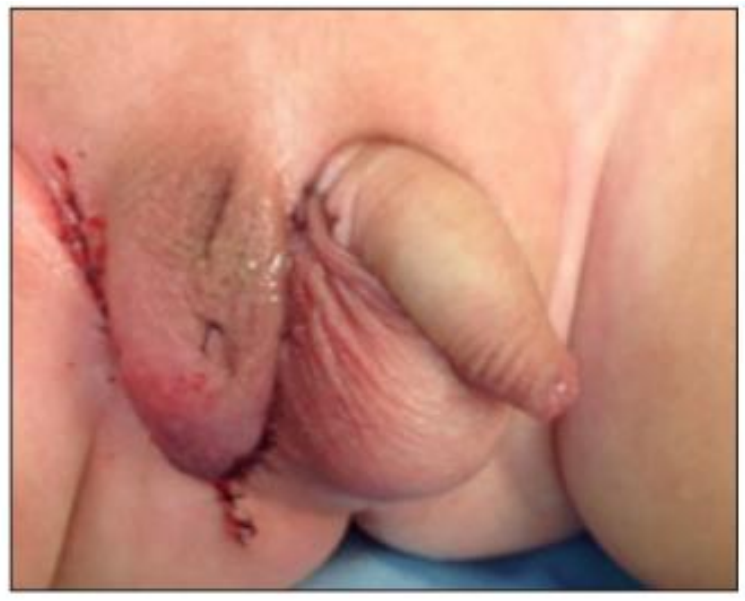

Fuente: (Córdova, Marín, Sánchez-Paredes, \& Borbolla-Sala, 2013).

Atendiendo a la localización de los testículos no palpables, se plantea la cirugía más adecuada para cada paciente. Las limitaciones de la ecografía, tomografía axial computarizada 


\section{Indicaciones de Orquidopexia en problemas testiculares en recién nacidos}

Vol. 3, núm. 3 Esp., (2019)

David Israel Cárdenas Acosta; Sandra Elizabeth Montufar Paguay

(TAC) y resonancia magnética demuestran que no existe un método de imagen ideal para localizar estos testículos. La laparoscopia fue utilizada inicialmente como herramienta diagnóstica para estos testículos no palpables con una mayor precisión que los métodos de imagen. Pero fue Jordan y cols, en 1992 los que introdujeron la aplicación terapéutica de la laparoscopia en varones con testes no palpables. Con los avances en la técnica e instrumentación, la Orquidopexia laparoscópica se ha convertido en el tratamiento de elección de los niños con testículos no palpables (Tejedor, y otros, 2008, pág. 191).

Para un teste no palpable la exploración laparoscópica es el "Gold Standard” y permite hacer diagnóstico y realizar descensos laparoscópicos en 1 o 2 tiempos según sea su cercanía al orificio inguinal profundo (OIP). De esta manera se recomiendan diversos algoritmos de conducta en el tratamiento de una criptorquidia. Sin embargo, en la práctica clínica, vemos que existe un grupo de pacientes con TND palpables de difícil resolución quirúrgica, en los que la liberación laparoscópica de los vasos espermáticos y el deferente en el retroperitoneo, facilitaría la Orquidopexia abierta en un tiempo y mejoraría el pronóstico de posición y vitalidad testicular a largo plazo (Sepúlveda, 2013, pág. 14).

\section{Metodología.}

La metodología de la investigación es de tipo revisión bibliográfica, para ello se ha recurrido a diferentes medios electrónicos, donde se han revisado trabajos de investigación similares que han estudiado todo lo referente a lesiones o condiciones testiculares en infantes y los métodos más utilizados o sugeridos para corregirlos. 


\section{Indicaciones de Orquidopexia en problemas testiculares en recién nacidos \\ Vol. 3, núm. 3 Esp., (2019) \\ David Israel Cárdenas Acosta; Sandra Elizabeth Montufar Paguay}

\section{Resultados.}

La localización ectópica más frecuente es la región perineal, seguida del conducto femoral, área púbica próxima al pene y finalmente hemiescroto contralateral. El $90 \%$ de las alteraciones escrotales; se asocian a hipospadias, cuerda o alteraciones del seno urogenital. Las tres anomalías escrotales encontradas fueron: el escroto bífido, transposición penoescrotal y el escroto ectópico o accesorio (Córdova, Marín, Sánchez-Paredes, \& Borbolla-Sala, 2013, pág. 97).

En un estudio realizado a 142 pacientes con diagnóstico de testículo no palpable, solo en el 9\% de los casos estudiados se recomendó realizar una Orquidopexia video-asistida sin sección de los vasos espermáticos testículo no palpable y los resultados fueron los siguientes:

- Los testículos fueron descendidos a la bolsa escrotal sin existir ninguna complicación intraoperatoria en el $100 \%$ de los casos. El tiempo quirúrgico osciló entre 40 y 80 minutos (media, 60 minutos). La primera revisión en consulta se realizó a la semana de la cirugía con posterior seguimiento entre 6 meses y 4 años (atendiendo a cada caso). La evolución ha sido satisfactoria en 11 de los pacientes, mientras que en los otros 2 se produjo un ascenso del testículo intervenido. Un Figura 1. Localización del teste criptorquídicos y sus elementos. Figura 2. Sección del gubernaculum distal. Figura 3. Descenso del testículo al escroto. Figura 4. Parche de peritoneo posterior entre vasos espermáticos y conducto deferente. Indicaciones, técnica y resultados de la orquidopexia laparoscópica en un solo tiempo 193 caso de Orquidopexia laparoscópica se asoció a la reparación de una hernia incarcerada (Tejedor, y otros, 2008, pág. 191). 


\section{Indicaciones de Orquidopexia en problemas testiculares en recién nacidos}

Vol. 3, núm. 3 Esp., (2019)

David Israel Cárdenas Acosta; Sandra Elizabeth Montufar Paguay

En un estudio realizado en el servicio de urología del Instituto Nacional de Pediatría, en donde se tomó una muestra de 60 pacientes, los resultados fueron los siguientes:

- El lado afectado más frecuente fue en forma bilateral en 23 pacientes (46\%), el lado izquierdo en segundo lugar con 18 pacientes (36\%) y el lado derecho con nueve pacientes $(18 \%)$

- En cuanto a la localización del testículo a nivel del canal inguinal en la exploración prequirúrgica se encontró que en 25 pacientes (50\%) se localizaron en el tercio medio del canal inguinal, en 16 pacientes (31\%) en el tercio superior del canal inguinal y en 10 pacientes (19\%) en el tercio inferior del canal inguinal (Herrera García, Cuevas Alpuche, Solorzano Morales, Aguilar Anzures, \& Olivera Vasquez, 2008).

En un estudio realizado en el Hospital de Especialidades de las Fuerzas Armadas de Quito, donde se revisaron las historias clínicas de 110 pacientes con diagnóstico de criptorquidia, los resultados más relevantes fueron los siguientes:

- La edad más frecuente de realización de la Orquidopexia en pacientes con criptorquidia atendidos en el servicio de Cirugía Pediátrica del HE-1 fue de 1 año 1 mes a 3 años con un número de 51 casos que corresponde al 46\%; en orden descendente tenemos a las edades de 5 años 1 mes a 10 años con 26 que corresponde al 24\%; luego continúan los niños < a un año con 11 casos correspondiente al 10\% y finalmente los niños de 10 años 1 mes a 15 años con un número de 7 casos correspondiente al $6 \%$.

- En la técnica quirúrgica de Bianchi del total de 69 pacientes, se determinó que 41 casos tuvieron un tiempo de duración en la cirugía de 30 minutos correspondiente al 59\%; 


\section{Indicaciones de Orquidopexia en problemas testiculares en recién nacidos}

Vol. 3, núm. 3 Esp., (2019)

David Israel Cárdenas Acosta; Sandra Elizabeth Montufar Paguay

mientras que en la técnica de Shoemaker el tiempo transcurrido en la duración de la cirugía predominó entre 31 a 45 minutos con 19 pacientes que representan el $46 \%$ (Chocho Tapia, 2013).

De enero de 2002 a septiembre del 2003 se intervino a 27 pacientes con 30 testículos no descendidos, valorados de manera inicial por su médico pediatra y con diagnóstico clínico confirmado por el cirujano o urólogo pediatra. En tres personas se presentó TND bilateral. A la exploración inguinoescrotal, 19 testículos fueron palpables y 11 no palpables. De los palpables, 9 (47.3\%) se localizaron en el tercio superior del canal inguinal, $4(21.1 \%)$ en el tercio medio y 6 (31.6\%) en el inferior. Trece TND fueron derechos y seis izquierdos. La edad a la que se operaron varió de ocho meses a nueve años, con un promedio de 21.7 meses. Todos se abordaron y operaron con la técnica de Orquidopexia laparoscópica descrita. El tiempo quirúrgico en ambos grupos fue en promedio de 50 min. En el grupo de TND palpables, todos se hicieron descender por laparoscopia, excepto uno en el cual el testículo era ectópico y se localizó por arriba de la aponeurosis del oblicuo mayor, lo que obligó a su conversión a orquidopexia abierta. En este grupo, cinco (26.3\%) fueron peeping testis y 13 (68.4\%) en el canal inguinal. Se identificó hernia inguinal indirecta en 15 casos (78\%) (Riquelme Heras, Landa Juárez, Miguel Gómez, Meneses Juárez, \& Andraca Dumit, 2009, pág. 217).

\section{Conclusiones.}

- En líneas generales la Orquidopexia evita problemas generados por la criptorquidia en niños mayores a 1 año de edad, como la torsión, facilita la exploración del testículo, sobre todo si se presenta un tumor testicular, mejora la función endocrina del mismo y crea un escroto con apariencia normal. 


\section{Indicaciones de Orquidopexia en problemas testiculares en recién nacidos}

Vol. 3, núm. 3 Esp., (2019)

David Israel Cárdenas Acosta; Sandra Elizabeth Montufar Paguay

- Es importante realizar la intervención quirúrgica antes de los 18 meses de edad, ya que preserva la función reproductiva y hormonal del testículo, pasada esa edad las alteraciones del tejido testicular pueden ser irreversibles.

- la técnica laparoscópica es la más utilizada y con mayores éxitos para la corrección de la criptorquidia, en la mayoría de los casos los testículos fueron descendidos a la bolsa escrotal.

- La Orquidopexia se presenta en durante el término de la gestación, lo que representa aproximadamente un $3 \%$, en lo que respecta a la criptorquidia las intervenciones quirúrgicas hay estudios que las han realizado entre 1 a los 3 años de edad.

\section{Bibliografía.}

Chocho Tapia, J. V. (2013). Orquidopexia, abordaje paraescrotal vs inguinal en pacientes con criptorquidea. Loja.

Córdova, J. E., Marín, A., Sánchez-Paredes, V., \& Borbolla-Sala, M. (2013). Escroto y testículo ectópico verdadero. Reporte de un caso. Salud en Tabasco, 19(3), 95-98.

Herrera García, W. E., Cuevas Alpuche, J., Solorzano Morales, S. A., Aguilar Anzures, R., \& Olivera Vasquez, J. L. (2008). Orquidopexia Paraescrotal en Niños con Criptorquidia Inguinal. Revista Mexicana de Cirugía Pediátrica, , 15(4), 161-168.

Herrera-García, W. E., Cuevas-Alpuche, J., Olivera-Vázquez, J., Aguilar-Anzures, R., \& Granados-Navas, F. (2009). Orquidopexia paraescrotal en pacientes con criptorquidia inguinal. Acta Pediátrica de México, 30(2), 89-93.

Neff, D. M. (s.f.). Orquidopexia laparoscópica. Obtenido de http://stjosepheureka.myhospitalwebsite.com/apps/healthgate/article.aspx?chunkiid=6295 09

Riquelme Heras, M. A., Landa Juárez, S., Miguel Gómez, D., Meneses Juárez, H., \& Andraca Dumit, R. (2009). Orquidopexia laparoscópica en el testículo no descendido, palpable y no palpable. Rev Mex Urol, 69(5), 215-218.

Sepúlveda, J. (2013). Orquidopexia videoasistida (OVA):¿ es la técnica de elección para una criptorquidia palpable difícil? Revista Chilena de Urología, 78(2). 
Indicaciones de Orquidopexia en problemas testiculares en recién nacidos

Vol. 3, núm. 3 Esp., (2019)

David Israel Cárdenas Acosta; Sandra Elizabeth Montufar Paguay

Tejedor, R., López, M., Cano, A., García, A., Cabezalí, D., Gómez, A., \& Aransay, A. (2008). Indicaciones, técnica y resultados de la orquidopexia laparoscópica en un solo tiempo. Cir Pediatr, 21, 191-194.

$$
\text { (C) }(1 \otimes(0)
$$

RECONOCIMIENTO-NOCOMERCIAL-COMPARTIRIGUAL

CC BY-NC-SA

ESTA LICENCIA PERMITE A OTROS ENTREMEZCLAR, AJUSTAR Y CONSTRUIR A PARTIR DE SU OBRA CON FINES NO COMERCIALES, SIEMPRE Y CUANDO LE RECONOZCAN LA AUTORÍA Y SUS NUEVAS CREACIONES ESTÉN BAJO UNA LICENCIA CON LOS MISMOS TÉRMINOS. 